\title{
Regional Nerve Block of the Temporomandibular Joint Capsule: A Technique for Clinical Research and Differential Diagnosis
}

\author{
IVEN KLINEBERG* and JOHN LILLIE
}

Department of Occlusion, School of Dentistry, University of Michigan, Ann Arbor, Michigan 48109 and Department of Anatomy, Medical School, University of Michigan, Ann Arbor, Michigan 48109

\begin{abstract}
In previous studies in which regional anesthesia of the temporomandibular joint capsule was used to examine the role of the joint in mandibular movement and distinguish it from muscle control, the anesthetic techniques used have not been satisfactorily described. The accuracy of the injection technique described in this paper was determined by dissection and radiographic examination of fixed and fresh specimens. Using this technique, trial patient studies were made using an anesthetic solution to which a radiopaque medium was added. Radiographic examination of the patients affirmed the location of the injected material, while clinical assessment determined its functional effectiveness. Using the described technique, anesthetic solution was accurately and reproducibly introduced posteriorly and laterally to the temporomandibular joint to achieve anesthesia of the joint.
\end{abstract}

J Dent Res 59(1 1):1930-1935, November 1980

\section{Introduction.}

Regional anesthesia has been employed in studying different aspects of articular mechanoreceptor control of jaw movement. These include studies of: mandibular kinaesthetic perception, ${ }^{14}$ mandibular border movements, 5 mastication, 6,7 tactile occlusal perception, 8 and swallowing. ${ }^{9}, 10$ Regional anesthesia has also been used prior to temporomandibular (TM) joint arthrography. 11-13

Despite the utility of TM joint capsule anesthesia to both the differential diagnosis of joint and occlusal dysfunction and basic masticatory research, a standard detailed description and evaluation of this anesthetic

Received for publication June 19, 1978

Accepted for publication April 11, 1980

This work was supported by Grant No. DE02731 .

*Present address: Department of Prosthetic Dentistry, The Dental School, University of Sydney, 2 Chalmers Stroet, Sydney N.S.W. 2010, Australia technique does not exist. As part of a systematic investigation of articular mechanoreceptor influences in motor control of jaw muscles in man, the technique reported in this paper was developed to ensure reliable regional articular nerve block of the TM articular capsule.

The innervation of the TM joint capsule in man is predominantly from the auriculotemporal branch of the mandibular division of the trigeminal nerve. Additional sensory supply from the mandibular division may pass to the capsule as sensory branches from the masseteric nerve and the deep temporal nerves. ${ }^{1,14}$ The majority of nerves and nerve endings of the capsule and disc are contained in the loose vascular connective tissues located posterior to the disc, connecting it with the posterior capsule, the retrodiscal pad.1,15-18 Branches from the masseteric nerve may innervate the lateral aspect of the capsule and associated TM ligament.

The technique described and assessed in this paper was designed to anesthetize the posterior and lateral region of the joint capsule and retrodiscal pad while avoiding the joint cavity and the innervation of the surrounding muscles of mastication. Surface landmarks for the injection were chosen, based on established dental prosthetic points and lines.

\section{Materials and methods.}

Pre-clinical studies $\$$ of the proposed injection technique were made using 12 preserved and two non-preserved human

$\$$ Human anatomic specimens for the preclinical studies were provided by the Department of Anatomy, School of Medicine, University of Michigan. 
specimens. A cream-thick mix of root canal cement (Roth cement $t^{\dagger}$ ) was prepared and injected into the specimens, using a $1 \frac{1 / 2}{2}$ inch, 20 gauge disposable needle. Volumes of .3 to $.5 \mathrm{ml}$ of the mix were injected into the periarticular tissues lateral and posterior to the articular capsule of the TM joint. The specimens were then left undisturbed for four $h$ at room temperature to allow the cement to set. The specimens were then radiographed using standard techniques, and the region was sequentially dissected and photographed. Direct visualization of the cement and the extent of its distribution in the tissues allowed accurate verification of the radiographic image-the correlative technique to be used in later patient studies.

Determination of the skin points for needle penetration in the preserved specimens was: 1) Posterior injection: This point was determined by measuring 10 to $12 \mathrm{~mm}$ anterior to the tragus of the ear along the tragal-lateral canthus line $(1$ to $3 \mathrm{~mm}$ posterior to the arbitrary transverse hinge axis skin point), and then inferiorly $5 \mathrm{~mm}$ from the point on the tragalcanthal line. This point was selected to achieve both an auriculotemporal nerve block and posterior periarticular injection. The needle was passed through the skin and directed posteroinferiorly five to ten degrees at this point to a depth of 15 $\mathrm{mm}$. For all injections described in this paper, deposition of the material was made as the needle was withdrawn. 2) Anterior injection: The skin point was determined by measuring $15 \mathrm{~mm}$ anterior to the tragus of the ear along the tragal-lateral canthus line and $15 \mathrm{~mm}$ below this point in the vertical plane. The needle was passed through the skin at this point to a depth of $10 \mathrm{~mm}$ and directed posteroinferiorly from 5 to 10 degrees.

In case of nonpreserved specimens, as a result of the variability encountered in the location of injected material in the preserved specimens, the anterior injection was omitted. The skin measurements and the technique for the posterior injection were not altered; however, by partially withdrawing the needle and then redirecting it

\footnotetext{
$\dagger$ Roth Drug Co., Chicago, IL
}

laterally from the initial site of penetration for the posterior injection, an effective lateral injection technique was developed. The lateral injection was carried out as follows: After the posterior injection and following almost complete withdrawal, the needle tip was repositioned at a point $5 \mathrm{~mm}$ anterosuperior to the original penetration point. The needle tip was thus located over the lateral region of TM capsule and lateral ligament. Injection of the material was begun at this point and continued during the withdrawal of the needle.

Trial clinical injections were then carried out, using a sterile technique on five subjects. A mixture $(50: 50)$ of iothalamate meglumine (a water soluble, radiopaque medium) with $2 \%$ xylocaine hydrochloride was injected. This mixture was sufficiently radiopaque to allow radiographic visualization of the material and to provide a subjective awareness of regional anesthesia.

Transcranial oblique and anteroposterior TM joint radiographs were taken immediately following periarticular injection to determine the location of the solution.

As a result of the pre-clinical studies, the clinical trial injections were performed in the manner previously described for injection of nonfixed specimens. A sterile technique was followed for each injection. In the case of the posterior injection, $1 \mathrm{ml}$ of the radiopaque-anesthetic solution was slowly injected as the needle was withdrawn. After the posterior injection, but before withdrawing the needle completely, the needle was redirected laterally in an anterosuperior direction for a distance of $5 \mathrm{~mm}$, and $0.5 \mathrm{ml}$ of solution was slowly injected during needle withdrawal. The anterior injection was omitted as before. Momentary thermal anesthesia of the skin prior to injection was obtained using a vapo-coolant spray, Fluoromethane. ${ }^{\circ}$ The ear canal and eye were protected from the spray.

\section{Results.}

Pre-clinical studies on preserved specimens. - The radiopaque material injected posterior to the TM joints was consistently found closely related to the posterior MO

+Conray $60^{\mathrm{R}}$, Mallinckrodt, Inc., St. Louis,

${ }^{\circ}$ Gebauer Chemical Co., Cleveland, $\mathrm{OH}$ 


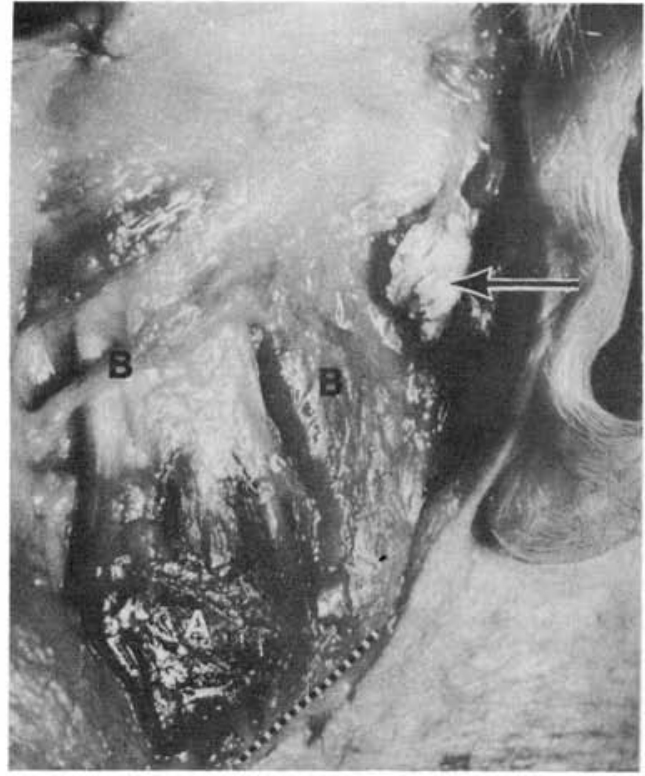

Fig. 1a

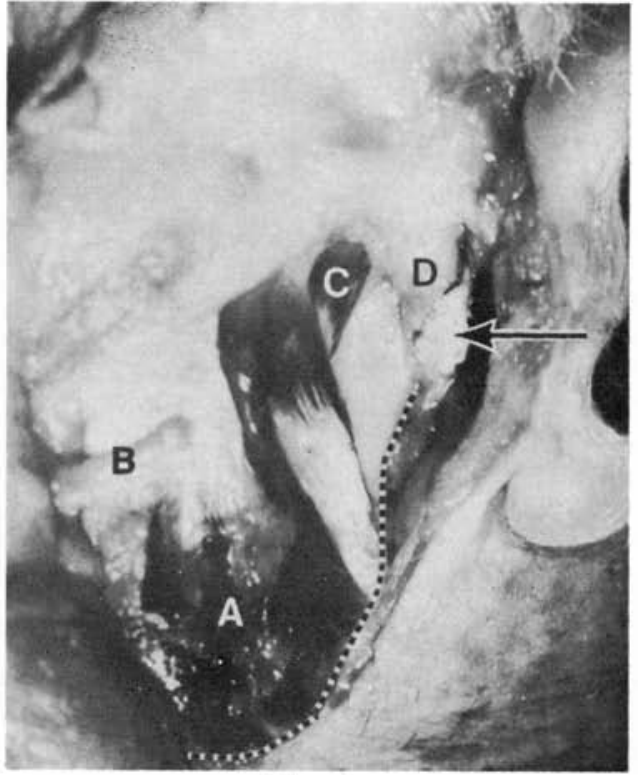

Fig. 1b

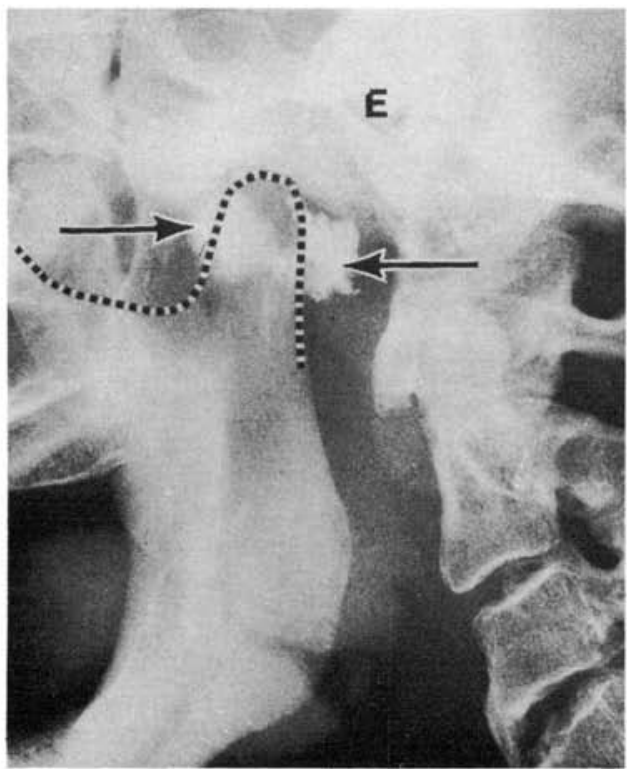

Fig. 1c

Fig. 1 - A representative dissection of a non-preserved specimen showing the superficial (a) and deep regions (b) and corresponding transcranial TM joint radiograph (c) of the injected material. In Fig. 1a, the site of deposition of the laterally-injected material is clearly shown lateral to the TM joint capsule. Fig. $1 \mathrm{~b}$ shows the location of the posteriorly-injected material. A transcranial radiograph (Fig. 1c) demonstrates the placement of (left arrow) laterally- and (right arrow) posteriorly-injected material. Both dissections and the radiograph show the proximity of lateral and posterior injection material to the corresponding periarticular areas. Labeled structures in Fig. 1 are: A) superficial head of the masseter muscle; B) parotid gland and duct; C) deep head of the masseter muscle; D) joint capsule of the temporomandibular joint; and E) external auditory meatus. Mandibular angle and condyle contours are noted by dashed lines. 
articular tissues. Though radiographs showed small variations in anteroposterior location of the material, dissection confirmed that these variations were parallax errors inherent in transcranial radiographic projections and that the material was actually closely related to the posterior articular tissue.

The anterior articular injections were found to be more variable, and transcranial radiographs and dissection showed that the injected material was placed anterior to the joint capsule. The injected material was consistently found overlying the lateral pterygoid muscle and on one occasion within the deep fibers of the masseter muscle.

The distance of the injected material from the lateral condylar tubercle was measured. With all posterior injections, the radiopaque material was located within a distance of $5 \mathrm{~mm}$ from the tubercle. With all anterior injections, the material was located at a distance greater than $5 \mathrm{~mm}$ from this condylar landmark.

Pre-clinical studies on non-preserved specimens. - The radiopaque material injected posterior to the TM joints was found close to the posterior periarticular tissues, and in no instance was material found in the joint cavity. Fig. 1 shows (a) laterally- and (b) posteriorly-injected material in apposition to the corresponding periarticular area; this is also depicted in the radiograph.

The non-preserved specimens indicated that there was little likelihood of the lateral injection affecting the lateral pterygoid or masseter muscles, since the injection site was considerably posterior and superficial to both areas of innervation.

The non-preserved specimens allowed a more precise injection of material and so provided more reliable pre-clinical data as a prelude to clinical injections.

Trial clinical injections. - Five trial clinical injections of radiopaque material were made. Radiographs showed the material to be located posterior and lateral to the periarticular regions. Fig. 2 shows representative radiographs. The three views on the left were taken before injection and those on the right after injection. The upper views (2a) are anteroposterior projections. In the right view radiopaque material (arrow) can be seen posterior and posteromedial to the periarticular region. The other two views are standard transcranial-oblique projections with teeth in intercuspal contact $(2 b)$ and the jaw open (2c). Radiopaque material can be seen in the posterior and lateral periarticular areas. The degree of opacity of the material relative to bone makes it best visualized when the jaw is open (2c).

In each trial clinical injection, the subject was aware of minimal or no discomfort during the placement of the material and subsequently experienced cutaneous anesthesia over the distribution of the auriculotemporal nerve and in the region immediately overlying the TM joint. Based on repeated subjective evaluation and radiographs, superficial anesthesia was well defined, and the injected material did not extend beyond the anterior region of the condyle.

\section{Discussion.}

Certain difficulties existed with injections into fixed specimens because of the inability to satisfactorily palpate the condylar head and periarticular tissues, the difficulty in penetrating the fixed tissues, and the displacement of the tissues by the embalming process. These features resulted in some variation in the placement of material during the pre-clinical studies of preserved specimens. These difficulties were overcome by the use of non-preserved specimens in preclinical studies. Studies on these specimens showed that it was possible to inject the lateral and posterior periarticular regions without directly affecting the surrounding muscles of mastication. Anterior injections frequently involved one or both heads of the lateral pterygoid muscle medially, and/or, more superficially, the fibers of the masseter muscle. In the clinical studies, no anterior injections were attempted, but posterior and lateral periarticular regions were consistently anesthetized, and radiographic confirmation indicated satisfactory distribution of solution.

This technique was designed to anesthetize the posterior and lateral articular nerves at their origin from the auriculotemporal nerve. The articular receptor population distribution in human ${ }^{1}$ and cat ${ }^{17,18}$ and the analyses of their fiber caliber have illustrated that the posterior articular capsule and nerve contain the greatest population density of receptors and articular nerve endings, respectively. The lateral capsule and 


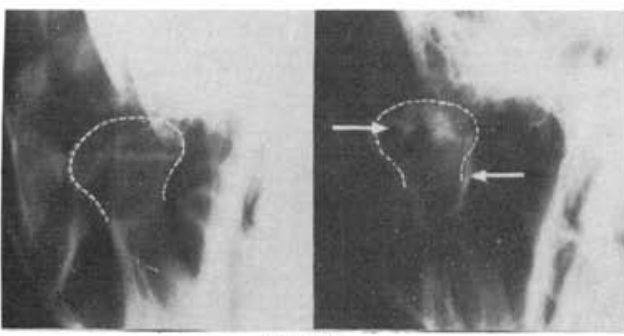

Fig. 2a

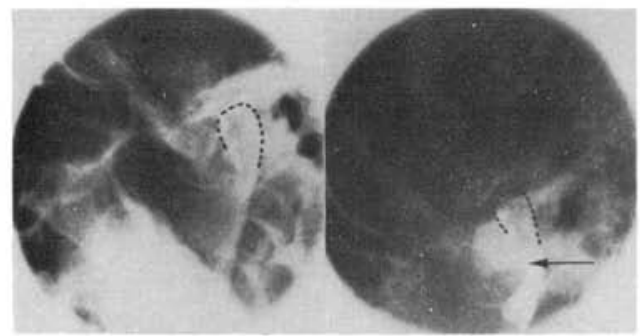

Fig. 2b

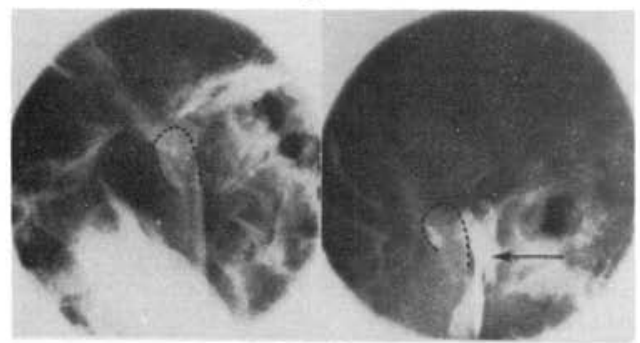

Fig. 2c

Fig. 2 - A representative series from the same TM joint of three sets of radiographs taken before (left) and after (right) a clinical injection of a mixture of Conray $60^{\mathrm{R}}$ and xylocaine. The radiopaque material is noted by the arrows in the right radiographs. Set a is an anteroposterior view and sets $b$ and $c$ are transcranial oblique views $\mathrm{b}$ with the jaw closed and $\mathrm{c}$ with the jaw open.

lateral ligament may be innervated by an independent articular branch or by filaments from the posterior nerve. 1,18 Generally, however, this region, as well as the anterior and medial capsule, are relatively sparsely innervated. Thus, the described regional nerve block of the posterior and lateral capsule should be sufficient to anesthetize the majority of articular nerve endings.

By recording jaw movements during mastication in patients before and after TM joint capsular anesthesia, the clinical effectiveness of this technique could be demon- strated. ${ }^{7,19}$ The results of these studies have been discussed in detail elsewhere, 7,19 but the studies showed that following capsular anesthesia there was a significant change in the spatial form of the functional envelope.

Concern for the other contents of the parotid gland fossa should be exercised. While the described injection technique when properly employed involves only the contents of the retroarticular portion of the fossa (the auriculotemporal nerve and a deep lobe of the parotid gland), with excessive anesthetic volumes or improper needle placement, other structures may be involved. Inferior placement at the level of the condylar neck may result in a hematoma from injury to the maxillary vein or an intraarterial injection of its vascular counterpart. Large injection volumes or superficial placement of the lateral injection may block the peripheral branches of the facial nerve (temporofacial division) and result in temporary paralysis of the orbicularis oculi muscle with attendant corneal drying.

\section{Conclusions.}

A technique has been described for regional nerve blockage of the articular capsule of the TM joint. The technique is based on correlative studies of preserved and non-preserved specimens and clinical applications. Radiography and appropriate dissection to localize the injected material confirmed the reliability of the technique. The utility of this technique in clinical studies on the motor control of jaw muscles and in differential diagnosis of orofacial pain is anticipated.

\section{REFERENCES}

1. THILANDER, B.: Innervation of the Temporomandibular Joint Capsule in Man, Trans $R$ Sch Dent 2:1-67, 1961.

2. RANSJO, K. and THILANDER, B.: Perception of Mandibular Position in Cases of Temporomandibular Joint Disorders, Odont T 71:134-144, 1963.

3. LARSSON, L.-E. and THILANDER, B.: Mandibular Positioning: The Effect of Pressure on the Joint Capsule, Acta Neurol Scand 40:131-143, 1964.

4. HELLSING, G.: Mandibular Kinaesthetic Illusions Induced by Vibration of Human Jaw Muscles, In press.

5. POSSELT, U. and THILANDER, B.: Influence of the Innervation of the Temporo- 
mandibular Joint Capsule on Mandibular Border Movements, Acta Odont Scand 23: 601-613, 1965 .

6. SCHAERER, P.; LEGAULT, J.V.; and ZANDER, H.A.: Mastication Under Anaesthesia, Helv Odont Act 10:130-134, 1966.

7. KLINEBERG, I. and ASH, M.M.: Some Temporomandibular Articular Reflex Effects on Jaw Muscles, IADR Progr \& Abst 57: No. 130,1978 .

8. CAFFESSE, R.G.; CARRARO, J.J.; and ALBANO, E.A.: Influence of Temporomandibular Joint Receptors on Tactile Occlusal Perception, J Periodont Res 8:400-403, 1973.

9. INGERVALL, B.; BRATT, C.M.; CARLSSON, G.E.; HELKIMO, M.; and LANTZ, B.: Positions and Movements of Mandible and Hyoid Bone During Swallowing: A Cineradiographic Study of Swallowing with and without Anesthesia of the Temporomandibular Joints, Acta Odont Scand 29: 549-562, 1971.

10. INGERVALL, B.; BRATT, C.M.; CARLSSON, G.E.; HELKIMO, M.; and LANTZ, B.: Duration of Swallowing with and without Anaesthesia of the Temporomandibular Joints Scand J Dent Res 80:189-196, 1972.

11. TOLLER, P.A.: Opaque Arthrography of the Temporomandibular Joint, Int $J$ Oral Surg 3:17-28, 1974.
12. TOLLER, P.A.: Non-surgical Treatment of Dysfunction of the Temporomandibular Joint, Oral Sci Rev 7:70-85, 1976.

13. WILKES, C.H.: Arthrography of the Temporomandibular Joint in Patients with the TMJ Pain-dysfunction Syndrome, Minn Med 61: 645-652, 1978.

14. SICHER, H. and DUBRUL, E.L.: Oral Anatomy, 5th ed., St. Louis: C.V. Mosby Co., 1970 , p. 173.

15. RAMFJORD, S. and HINIKER, J.: Distal Displacement of the Mandible in Adult Rhesus Monkeys, J Prosthet Dent 13:491-502, 1966.

16. SICHER, H.: Structure and Functional Basis for Disorders of the Temporomandibular Joint, I Oral Surg 13:275, 1965.

17. GREENFIELD, B.E. and WYKE, B.D.: Reflex Innervation of the Temporomandibular Joint, Nature 21 1:940-941, 1966.

18. KLINEBERG, I.: Structure and Function of Temporomandibular Joint Innervation, $A n n$ $R$ Coll Surg Engl 49:268-288, 1971.

19. KLINEBERG, I.: Influences of Temporomandibular Articular Mechanoreceptors on Functional Jaw Movements, $J$ Oral Rehabil, In press, 1980.

\section{NOW AVAILABLE}

During the AADR Annual Session in Los Angeles this past March, the Committee on Health Promotion sponsored a symposium called "Promoting the Use of Fluorides in Communities: Past Accomplishments and Future Perspectives." The program featured presentations by six noted authorities in various aspects of community fluoridation.

The proceedings of this Symposium are now available in the Journal of Public Health Dentistry, Vol. 40, No. 3, Summer, 1980. If you are interested in obtaining a copy, please contact Dr. Alice M. Horowitz, National Caries Program, NIH/NIDR, Bethesda, MD 20205. 